\title{
Electrode Placement Strategies for 3D EIT
}

\author{
B.M. Graham, A. Adler \\ School of Information Technology and Engineering (SITE), University of Ottawa, Canada
}

\begin{abstract}
We test strategies for placing EIT electrodes on a 3D medium for the purpose of calculating 3D reconstructions using clinical equipment intended for use with a $2 \mathrm{D}$ adjacent drive protocol. The goal of this work was to compare seven such strategies in order to determine if any were clearly superior to the others for clinical applications. For the limited set of strategies investigated, none were significantly better than the others in terms of performance under ideal conditions. However, when noise and electrode placement errors were considered the Planar Electrode Placement Strategy emerged as a recommended strategy for clinical use.
\end{abstract}

\section{INTRODUCTION}

EIT attempts to calculate a stable and accurate image of the conductivity or conductivity change within a medium from which electrical measurements are made on the medium boundary. Electrode placement for 2D reconstruction algorithms is confined to planar arrangements that match the $2 \mathrm{D}$ reconstruction geometry. However the EIT problem is inherently 3D as currents cannot be confined to flow in the plane. Consequently $2 \mathrm{D}$ reconstructions are subject to artifacts generated by off plane contrasts. Compared to 2D there are many more ways to arrange and sequence electrodes when placing them in 3D. In this paper we investigate seven 3D electrode placement (EP) strategies and evaluate their performance in terms of several figures of merit, immunity to noise, and performance in the presence of electrode placement errors.

\section{METHODS}

We consider EIT difference imaging, in which a small change in conductivity, $\mathbf{x}=\boldsymbol{\sigma}_{2}-\boldsymbol{\sigma}_{1}$ results in a change in difference signal, $\mathbf{z}=\mathbf{v}_{2}-\mathbf{v}_{1}$. This is linearized as $\mathbf{z}=\mathbf{H} \mathbf{x}+\mathbf{n}$ for small changes around a homogenous background conductivity where $\mathbf{H}$ is the Jacobian and $\mathbf{n}$ is the measurement system noise. Tto overcome the illconditioning, eqn (1) is solved using the regularized inverse

$$
\hat{\mathbf{x}}=\left(\mathbf{H}^{\mathrm{T}} \mathbf{W H}+\lambda^{2} \mathbf{R}\right)^{-1} \mathbf{H}^{\mathrm{T}} \mathbf{W} \mathbf{z}=\mathbf{B z}
$$

where $\hat{\mathbf{x}}$ is an estimate of the change in conductivity distribution, $\mathbf{R}$ is a regularization matrix, $\lambda$ is a scalar regularization hyperparameter, and $\mathbf{W}$ models the system noise. We assume that all measurements have equal noise variance, thus $\mathbf{W}$ becomes a multiple of the identity matrix.
Eq (2) is solved using the Nodal Inverse Solver algorithm and regularization parameter selection of [4]. This algorithm developed with the EIDORS [2] package using the complete electrode model [6].

\section{A. EP Strategies}

Simulated data were generated from the 28 layer, 86016 element, 15805 node FEM mesh. Reconstructions were performed on the 28 layer, 21504 element, 4205 node FEM. Both meshes were designed to model a $28 \mathrm{~cm}$ diameter by $28 \mathrm{~cm}$ high cylindrical tank phantom from which we acquired test data using a 16 electrode, adjacent drive GoeMF II tomography system (Viasys Healthcare, Höchberg, FRG). Electrodes were arranged in two parallel plane arrangements; the aligned arrangement in which electrodes in each plane are vertically aligned and the offset arrangement in which the lower electrode plane is rotated such that the electrodes are offset by half the inter-electrode spacing. Two planes of electrodes lead to a logical partitioning of the tank into three zones (top and bottom end zones, and the middle zone). Electrodes that are arranged in either the aligned or offset arrangement can be connected to the equipment with various sequences. We call the combination of electrode arrangement and sequencing an Electrode Placement (EP) strategy. In this work we propose and evaluate seven EP strategies: Planar, Planar-Offset, Planar-Opposite, Zigzag, Zigzag-Offset, Zigzag-Opposite, and Square.

For the three Planar EP strategies, measurements are mainly taken between electrodes in the same plane (intraplanar), with the exception of measurements taken between electrodes $8 \& 9$, and $16 \& 1$ which are inter-planar measurements. With the three Zigzag patterns measurements are always taken between electrodes in different planes (inter-planar). The square EP strategy has an equal amount of data taken from inter- and intra-planar electrode pairs.

\section{B. Evaluation Procedure}

The EP strategies were initially evaluated using a set of data simulated using the larger FEM. The data set consisted of an impulse contrast located halfway along the radius of the tank $(\mathrm{r} / 2)$ that is moved through 28 vertical positions. Subsequently 28 reconstructions were calculated for each of 
the seven EP strategies under various conditions of noise and electrode placement errors. Reconstructions were evaluated and compared based on the following criteria:

- Resolution: BR ([4]) calculates the volume fraction of the elements that contain the largest amplitude contributions to $50 \%$ of total image amplitude. The set of elements that contribute to the blur radius is called the half amplitude (HA) set.

- Radial Position Error: (PE) is the normalized difference in radial position of the centre of mass of the reconstructed image $\mathrm{HA}$ set and the centre of mass of the generating impulse contrast. A negative PE indicates the reconstructed image is closer to the centre.

- Vertical PE: is the normalized difference in the vertical position of the centre of mass of the reconstructed image HA set and the centre of mass of the generating impulse contrast. A negative value indicates that reconstructed image is closer to the central plane.

- Image power: is the sum of the volume-weighted element conductivity amplitudes squared.

- Qualitative Evaluation of reconstructed images, which is primarily a subjective evaluation of image artifacts.

- Immunity to Noise.

- Immunity to systematic electrode placement errors.

\section{RESULTS}

3D Electrode placement strategies are compared for the given criteria.

\section{Evaluation of Maximum Performance Experiments}

The initial evaluation looked at the best case performance of the EP strategies (with no added simulation noise), giving the following observations:

- Resolution: all EP strategies vary as a function of the height of the contrast (figure 1). The Planar EP strategy has the best resolution in the end zones, the opposite strategies have the worse performance in the middle zone, however the differences are not significant and it appears that Resolution is not a strong discriminator of EP strategies.

- Vertical PE: Figure (2) shows reconstructed height vs true height. All the EP strategies suffer from a vertical range compression in the end sections. The Zigzag, ZigzagOffset, Square, and Planar-Opposite EP strategies have large non-linearity in the central region of the graph. This is undesirable as a small vertical movement of the generating contrast can cause a large change in the vertical position of the reconstruction. We consider Vertical PE to be a useful discriminator of EP strategies.

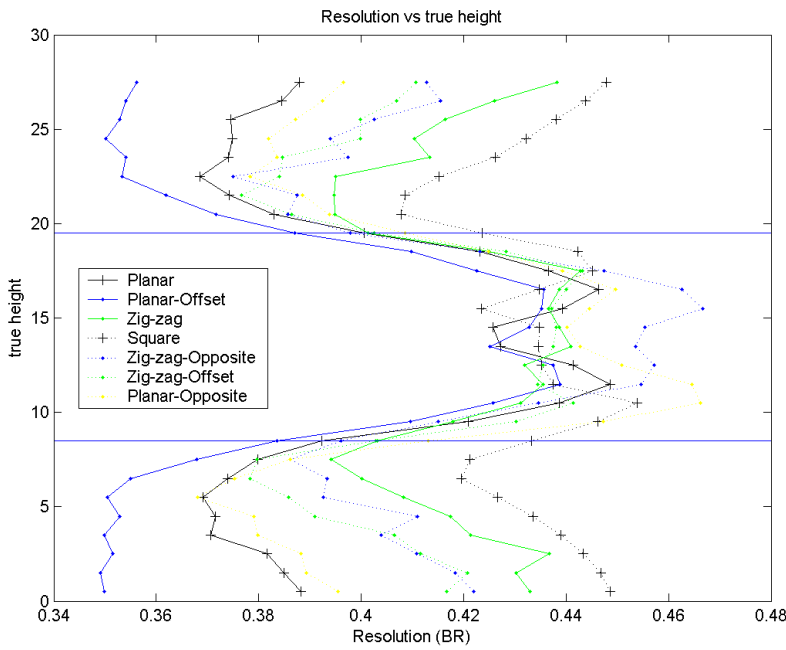

Figure 1. Resolution vs. target height

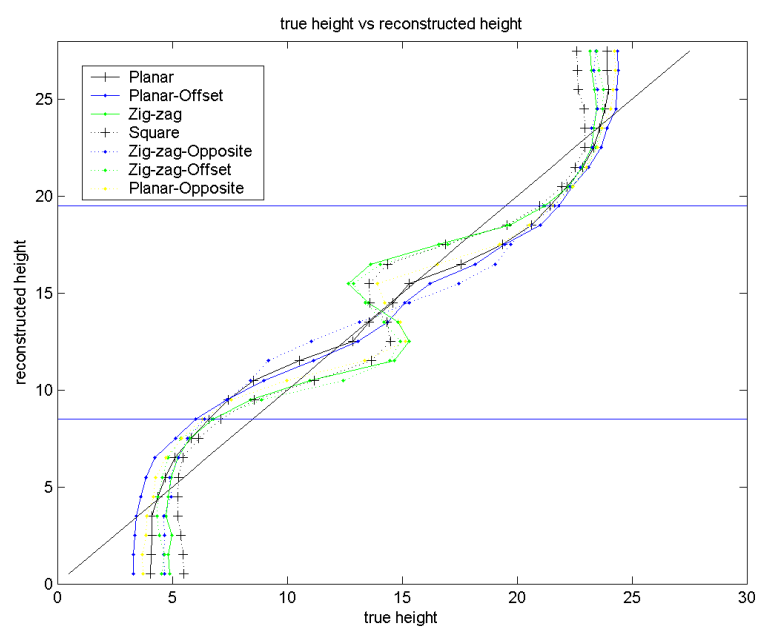

Figure 2. Vertical Position Error vs. target height

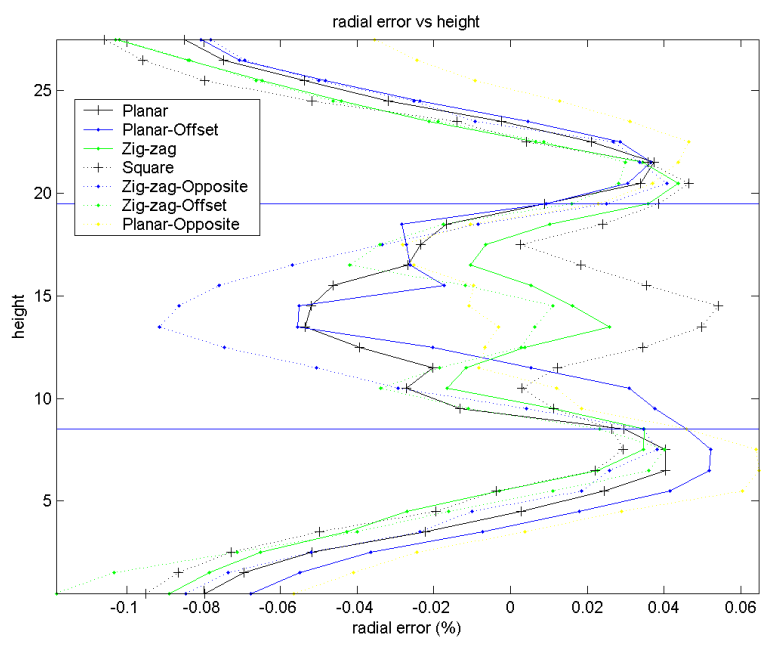

Figure 3. Radial Position Error vs. target height 
- Radial PE: Figure (3) shows that EP is worst when the contrast is far from the electrode planes. Results differ between EP strategies between electrode planes. Radial $\mathrm{PE}$ is not a strong discriminator of EP strategies.

- Image Power: A plot of Image Power vs true height shows that image power increases as the contrast location moves from the ends of the tank toward the electrode planes. The behaviour of the various EP strategies in the middle section is different for each strategy. We consider the Planar EP strategy to be slightly better than the other strategies in terms of the stability throughout the middle section. Overall, Image Power is not a strong discriminator of EP strategies.

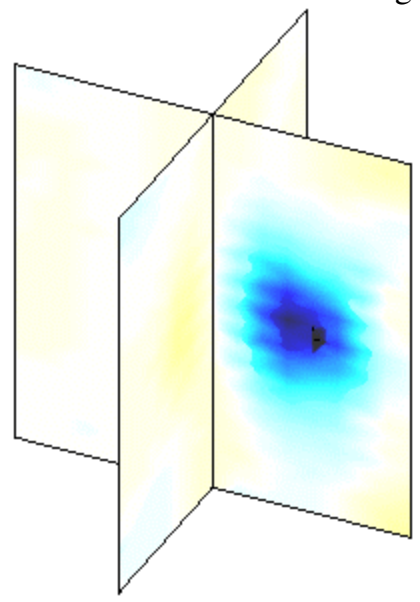

Figure 4 (a) Planar EP

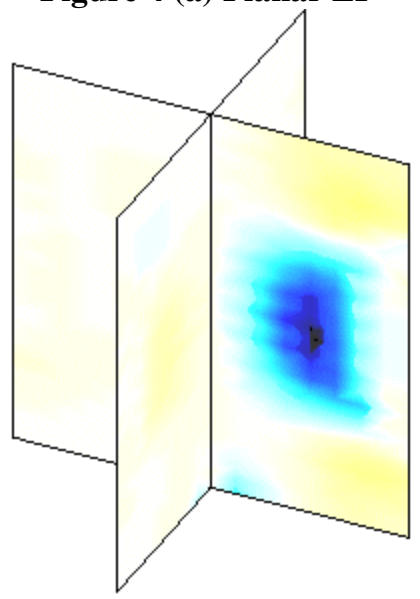

Figure 5 (a) Zigzag EP

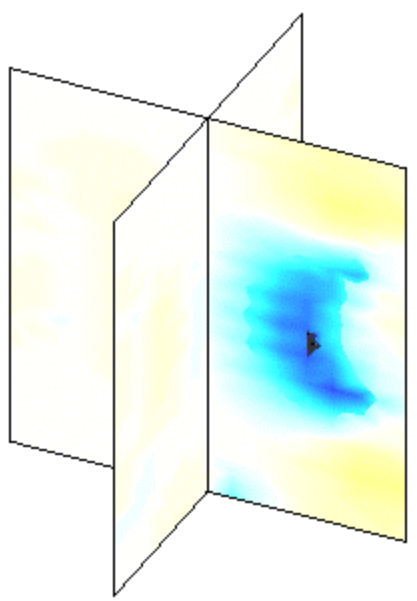

(b)Zigzag-Offset EP

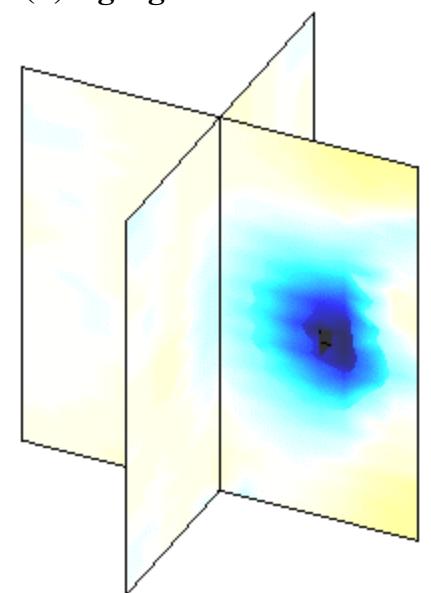

(b)Zigzag-Opposite EP

- Qualitative Evaluation: Figures 4 and 5 show images of contrasts at the centre plane of the tank. The Planar, Planar-Opposite, Planar-Offset and Square EP strategies produce spherical shaped reconstructions. The Zigzag and Zigzag-Opposite strategies produce vertically elongated images, while the Zigzag-Offset EP strategy, fig 4(b),

shows a "banana" shaped image. The best performance for contrasts in the end sections is with the Zigzag and Square EP strategies. The Planar, Planar-Offset (similar) and Planar-Opposite EP strategies produce large artifacts. In cases where the region of interest (ROI) is confined to the middle zone, it may be preferable to use an EP strategy that works very well in the ROI despite producing artifacts for contrasts located in the end zones.

\section{Evaluation of Noise Effects}

In addition to the baseline reconstructions discussed above, an additional six sets of reconstructions were calculated for each of the seven EP strategies in which AWG noise was added in six steps from $0.1 \%$ to $0.6 \%$. The Zigzag and Zigzag-Offset EP strategies failed for noise levels above $0.2 \%$ and the Square EP strategy failed for noise above $0.3 \%$. Useful reconstructions could be calculated using the two Opposite EP strategies with up to $0.6 \%$ noise, but with degraded Resolution and PE performance. The Planar and Planar-Offset EP strategies were very robust to noise; resolution and PE degraded slowly and good images were reconstructed with noise in excess of $0.6 \%$.

\section{E. Electrode Position Errors}

Two techniques were used to evaluate electrode position errors: 1) reconstructions were performed with a systematic electrode position error in which data collected with one of the EP strategies were reconstructed using the same electrode sequence but with the lower plane of electrodes rotated by half the inter-electrode distance (Offset Error). For example data generated with a Planar EP strategy was reconstructed using the Planar-Offset EP strategy. 2) a Layer Separation Error was simulated in which the distance between the electrode planes was increased from $11 \mathrm{~cm}$ (correct separation) to $20 \mathrm{~cm}$.

Offset Error: All EP strategies showed degraded resolution with the Zigzag-Offset pattern being the most adversely affected. The Planar-Opposite EP strategy gave the worst overall performance: a conductivity decrease gave images of a conductivity increase. The Planar, PlanarOffset, and Zigzag EP strategies were able to reconstruct a circular/spherical image without introducing image shape artifacts. However, in all cases the centre of mass of the reconstructions was rotated in the axial plane by about $20^{\circ}$. For functional imaging applications this rotation may be less significant if the magnitude of the conductivity change is accurate.

Layer separation error: Radial PE, Vertical PE and image power were not significantly affected by electrode plane errors for any of the EP strategies. However, all 
tragedies produced vertically elongated images with the Square and the two Opposite EP strategies being most affected, Zigzag and Zigzag-Offset strategies less so, and the Planar and Planar-Offset EP strategies the least. The Planar and Planar-Offset EP strategies also showed an increased Radial PE due to the contrast being pushed toward the tank centre for phantoms located in the end sections.

For contrasts located in the end zones, the Zigzag, Zigzag-Offset, and Square strategies show a "swirling artifact" while the Opposite EP strategies show an extensive vertical lengthening of the reconstructed contrast. Both the Planar and Planar-Offset EP strategies show little degradation due to electrode plane separation errors of up $6 \mathrm{~cm}$ on the $28 \mathrm{~cm}$ tall tank. The Planar-Offset is slightly more robust than the Planar EP strategy in this regard.

\section{F. Summary of Significant Observations}

The Planar EP strategy produces the largest signal, and produces circular/spherical reconstructions for contrasts located in the middle section. Compared to the other strategies it is the most robust to AWGN, is robust to layer separation errors, and is little affected by the offset error in that the image is rotated slightly about the vertical axis. Its worst performance is for contrasts located in the end sections where reconstructions are radially elongated. The Planar-Offset strategy is similar to the Planar EP strategy in that it produces circular/spherical reconstructions for contrasts located in the middle section, is robust to noise, and is only affected by the offset error in that the image is rotated slightly about the vertical axis. It is more resilient to layer separation errors than the Planar strategy, however it suffers from large swirling artifacts in the end sections. The Planar-Opposite strategy produces a circular/spherical reconstruction for contrasts in the middle section, but exhibits artifacts extending from the electrode plane to the image. This strategy is severely handicapped by its poor noise performance and the instability in VPE for contrasts near the central plane. The Zigzag strategy is highly susceptible to noise, and suffers from artifacts similar to those of the Planar-Opposite EP strategy for contrasts in the middle section.

The Zigzag-Offset strategy produces the best looking images for contrasts located in the end sections but produces non-circular/spherical images for central contrasts. This method also suffers from the instability in VPE, and shows artifacts extending from the electrode planes to the reconstructed image for contrasts in the middle section. Moreover, the Zigzag-Offset strategy has poor noise immunity. The Zigzag-Opposite strategy is able to produce images in the presence of AWGN; however the resolution and PE performance rapidly degrades. This strategy also suffers from the electrode to contrast artifacts for central contrasts. The Square strategy suffers from the instability in VPE, has poor noise performance, and shows the electrode to contrast artifacts.

\section{Conclusion}

This paper has investigated the performance of electrode placement strategies for a 16 electrode adjacent drive system. The current results suggest that no one EP strategy offers a dramatic improvement over the others under ideal conditions. Only when noise and electrode placement errors are considered does the choice of EP strategy become important. We make these observations:

- Opposite EP strategies are highly susceptible to corruption by noise.

- The Zigzag-Offset EP strategy is susceptible to Offset error.

- The Planar and Planar-Offset EP strategies are most robust to noise and systematic electrode placement errors.

- The Planar EP strategy provides the largest image power for contrasts located in the centre section.

The difficulty of placing electrodes accurately in vivo may be the largest discriminating factor amongst EP strategies intended for clinical use. Moreover electrode placement errors would be exacerbated due to subject movement. This leads one to prefer an EP strategy that is robust to electrode placement errors. Considering these factors, overall, we recommend Planar EP strategy.

\section{REFERENCES}

1. Adler A and Guardo R 1996 Electrical impedance tomography: regularised imaging and contrast detection. IEEE Trans. Med. Imaging 15 170-9

2. Adler A and Lionheart WR, 2006 Uses and abuses of EIDORS: An extensible software base for EIT, Physiol. Meas. 27:S25-S42, 2006

3. Cheney M, Isaacson D, Newell JC, Simske S and Goble JC 1991 NOSER: an algorithm for solving the inverse conductivity problem. Int. J. Imaging Syst. Technol 2 66-75

4. Graham BM and Adler A 2006 A Nodal Jacobian Based Algorithm for Reduced Complexity EIT Reconstructions. In press

5. Vauhkonen M, Kaipio JP, Somersalo E and Karjalainen PA 1997 Electrical impedance tomography with basis constraints. Inverse Problems 13 523-30

Address of the corresponding author:

Author: Brad Graham

Institute: University of Ottawa,(SITE)

Street: $\quad 161$ Louis Pasteur

City: Ottawa

Country: Canada

Email: $\quad$ graham.bm@sympatico.ca 